\title{
End Calorimeter Support Carriage Analysis
}

Engineering Note \# 3740.224-EN-285

Issued: February 26, 1991

Originator: Kay Weber/Rick Luther 
D-0 Engineering Note

3740.224-EN-285

Issued February 26,1991

\section{END CALORIMETER SUPPORT CARRIAGE ANALYSIS}

Kay Weber/Rick Luther

\section{INTRODUCTION}

The design of the EC carriage is based on the design of the CC carriage documented in D-0 Engineering Note 3740.215-EN-14. The non-symmetric weight distribution of the EC cryostat results in uneven loading of the arms of the EC carriage. The design load on each rear arm is $220,000 \mathrm{lb}$, while each front arm supports $40,000 \mathrm{lb}$. (The CC carriage design load was 200,000 lb on all four arms.) ANSYS finite element models were made representing various combinations of plate thicknesses in an attempt to balance the deflections of the arms. In the final design a 3/4" curved top plate is used with an additional 1.75" thick "doubler plate" incorporated into the rear arms. The bottom flanges are $2 "$ and $3 / 4$ " for the rear and front arms, respectively. Web plates are $3 / 4^{\prime \prime}$ for all arms. The carriage bottom plate is $2^{\prime \prime}$ thick, and other details are similar to the CC design.

\section{SUMMARY OF RESULTS}

Table 1 presents stress levels at various locations in the carriage along with allowable values taken from the AISC Specification for Structural Steel. Locations are shown in Figure 1. All calculations are contained in Appendix A.

\section{WELDS}

All welds in the structure are full-thickness groove welds or fillet welds made with E308/E309 filler metal having a minimum ultimate strength of $80 \mathrm{ksi}$. Fillet weld sizing calculations are included in Appendix A.

\section{DEFLECTIONS}

Table 2 gives deflections of the arms of the carriage for various combinations of plate thicknesses as determined by finite element analysis. Deflections of both carriages were measured during load tests and are documented in Engineering Note 3740.224-EN-282.

\section{FINITE ELEMENT ANALYSIS}

The finite element analysis used to size components of the carriages is described in Appendix B.

$$
\text { sh. } 1 \text { of } 5
$$


TABLE 1

STRESS SUMMARY

\begin{tabular}{|c|c|c|}
\hline Location & Stress (ksi) & Allowable (ksi) \\
\hline $\begin{array}{l}\text { Rear Arms } \\
\text { Section A-A } \\
\text { B-B } \\
\text { B5-B5 } \\
\text { C-C } \\
\text { D-D } \\
\text { E-E } \\
\text { Front Arms } \\
\text { Section A-A } \\
\text { B-B } \\
\text { B5-B5 } \\
\text { C-C } \\
\text { D-D } \\
\text { E-E }\end{array}$ & $\begin{array}{l}\mathrm{fb}=8.0 \\
\mathrm{fb}=3.8 \\
\mathrm{fb}=12.9 \\
\mathrm{fb}=11.3 \\
\mathrm{fb}=11.2 \\
\mathrm{ft}=17.0 \\
\mathrm{fc}=13.9 \\
\mathrm{fb}=2.4 \\
\mathrm{fb}=3.8 \\
\mathrm{fb}=5.6 \\
\mathrm{fb}=4.5 \\
\mathrm{fb}=5.1 \\
\mathrm{ft}=11.0 \\
\mathrm{fc}=2.7\end{array}$ & $\begin{array}{l}\mathrm{Fb}=19.8 \\
\mathrm{Fb}=19.8 \\
\mathrm{Fb}=19.8 \\
\mathrm{Fb}=19.8 \\
\mathrm{Fb}=18.0 \\
\mathrm{Ft}=18.0 \\
\mathrm{Fc}=18.0 \\
\mathrm{Fb}=19.8 \\
\mathrm{Fb}=19.8 \\
\mathrm{Fb}=19.8 \\
\mathrm{Fb}=19.8 \\
\mathrm{Fb}=18.0 \\
\mathrm{Ft}=18.0 \\
\mathrm{Fc}=18.0\end{array}$ \\
\hline $\begin{array}{l}\text { Stanchion Support } \\
\text { Plate Bending } \\
\text { Gusset Bending } \\
\text { Support Bracket } \\
\text { Web Bending } \\
\text { Shear } \\
\text { Plate Bending }\end{array}$ & $\begin{array}{c}\mathrm{fb}=12.6 \\
\mathrm{fs}=2.4 \\
\mathrm{fb}=13.5\end{array}$ & $\begin{array}{l}\mathrm{Fb}=19.8 \\
\mathrm{Fs}=12.0 \\
\mathrm{Fb}=22.5\end{array}$ \\
\hline
\end{tabular}

\section{ALLOWABLE STRESSES}

Allowable stresses for the carriage design were taken from Section 1.5 of the AISC Specification for Structural Steel for Buildings. The carriages are constructed of type 304 stainless steel having a minimum yield strength of $30 \mathrm{ksi}$ and a minimum ultimate strength of $75 \mathrm{ksi}$. The applicable allowable stresses are:

Bending in a compact section Bending in a general section Bending in a solid rect.angle Tension Compression (no buckling) Shear

$$
\begin{aligned}
& \mathrm{Fb}=.66 \mathrm{Fy}=19.8 \mathrm{ksi} \\
& \mathrm{Fb}=.60 \mathrm{Fy}=18.0 \\
& \mathrm{Fb}=.75 \mathrm{Fy}=22.5 \\
& \mathrm{Ft}=.60 \mathrm{Fy}=18.0 \\
& \mathrm{Fc}=.60 \mathrm{Fy}=18.0 \\
& \mathrm{Fs}=.40 \mathrm{Fy}=12.0
\end{aligned}
$$


ANSYS $4.3 \mathrm{~A}$

AUG 91989

105055

PLOT NO. 3

PREPT ELEMENTS

$\mathrm{ZV}=1$

$D I S T=85.626$

$Y F=1.001$

$Z F=-30.063$

PRECISE HIDDEN

Figure 1 
TABLE 2

EC CRADLE DEFLECTIONS

CENTER OF

FRONT ARM

CENTER OF

REAR ARM

1.5" TOP PLATE

$2^{n}$ ANGLE PLATES

$-.05728$

$-.2875$

1" FRONT, 2" REAR

TOP PLATE, 2" ANGLE

PLATES

$-.06337$

$-.2684$

1" FRONT, 2" REAR

TOP PLATE, 1" FRONT

ANGLE PLATE

$-.07431$

$-.2687$

1" FRONT, 2.5" REAR

TOP PLATE, $.75^{\prime \prime}$ FRONT

ANGLE PLATE

$-.07867$

$-.2521$

.75" FRONT, 2.5" REAR TOP PLATE, .75" FRONT ANGLE PLATE

$-.08629$

$-.2537$ 
sh 5 d 5
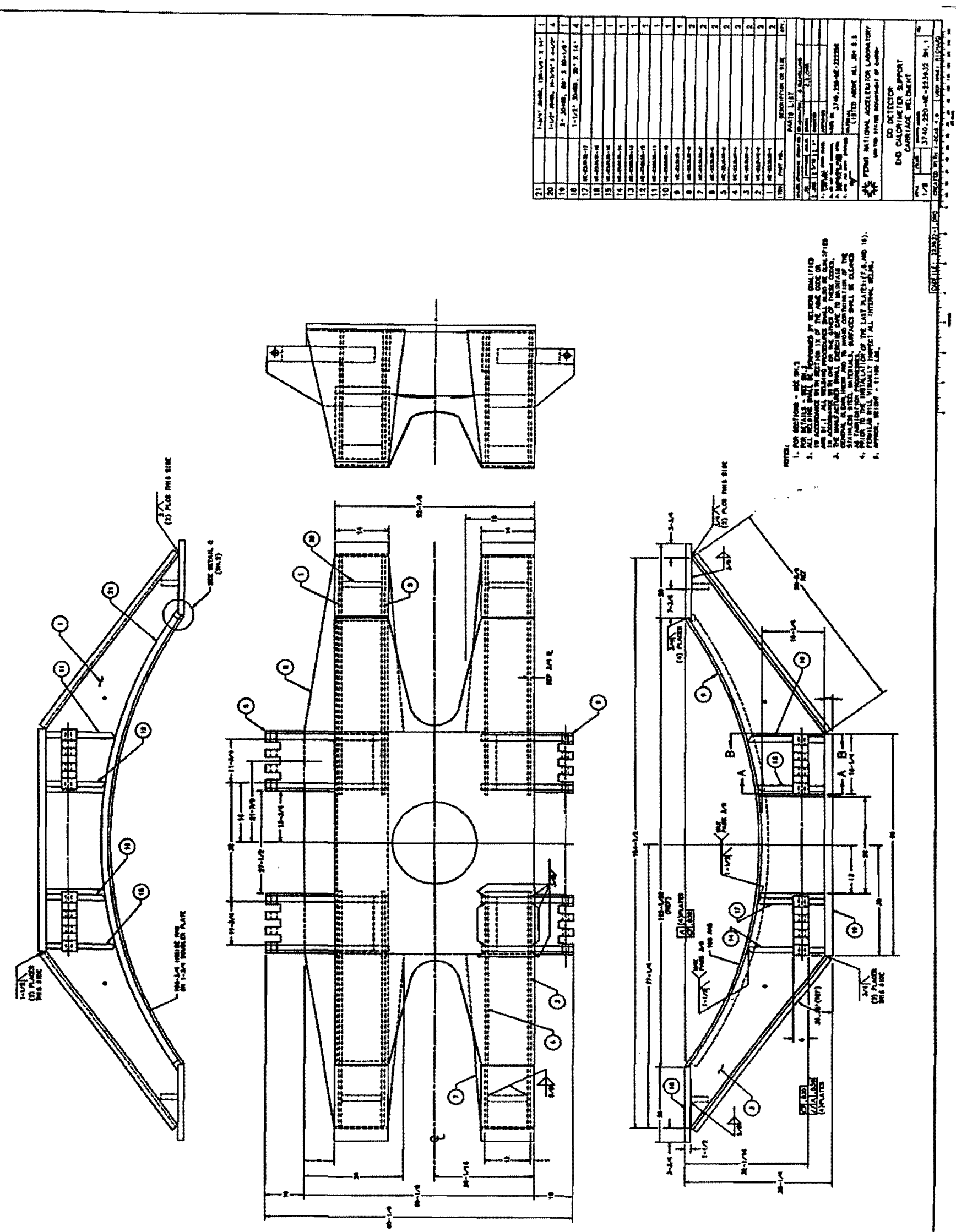


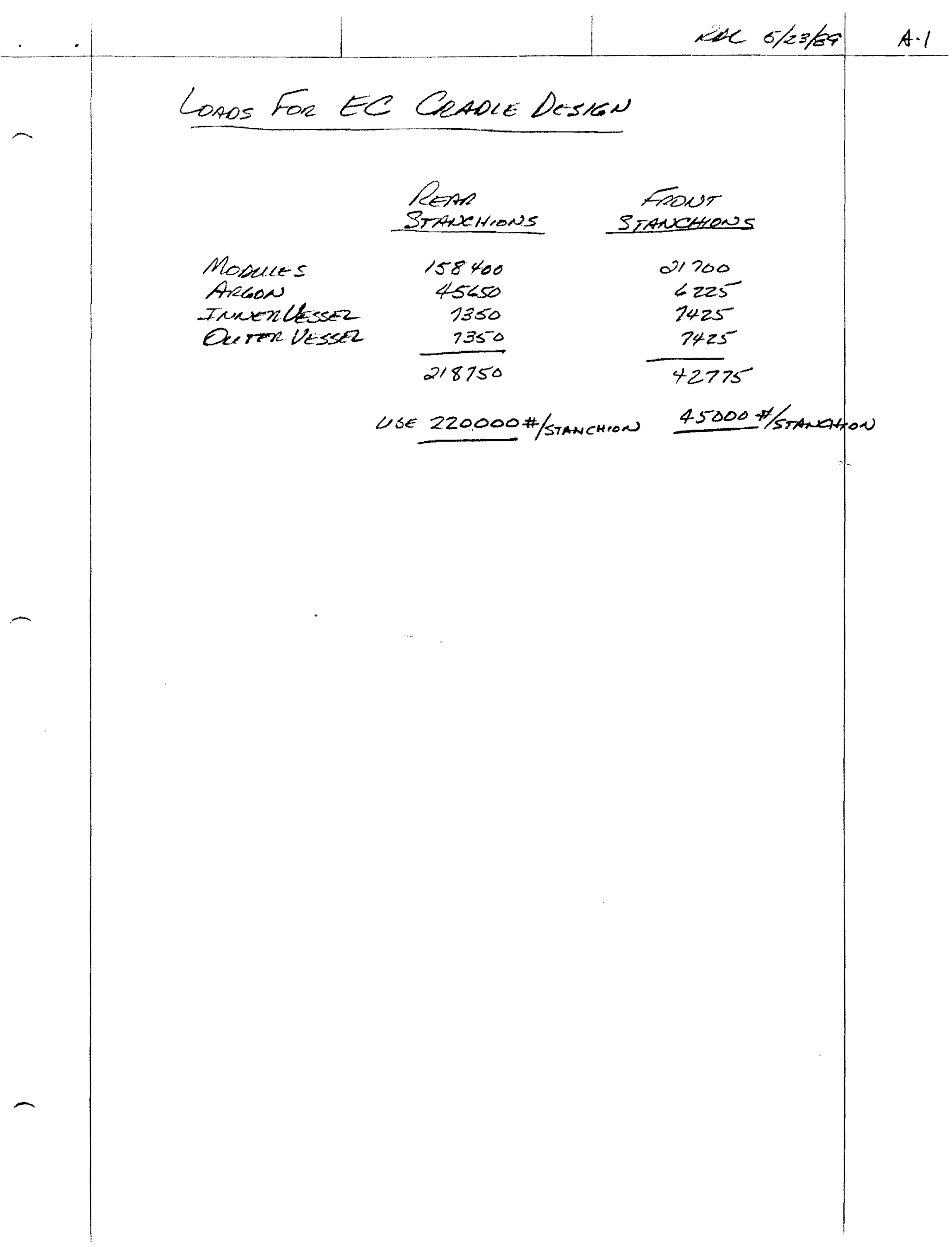




\section{SECTION A-A}

\section{REAR}

FRONT

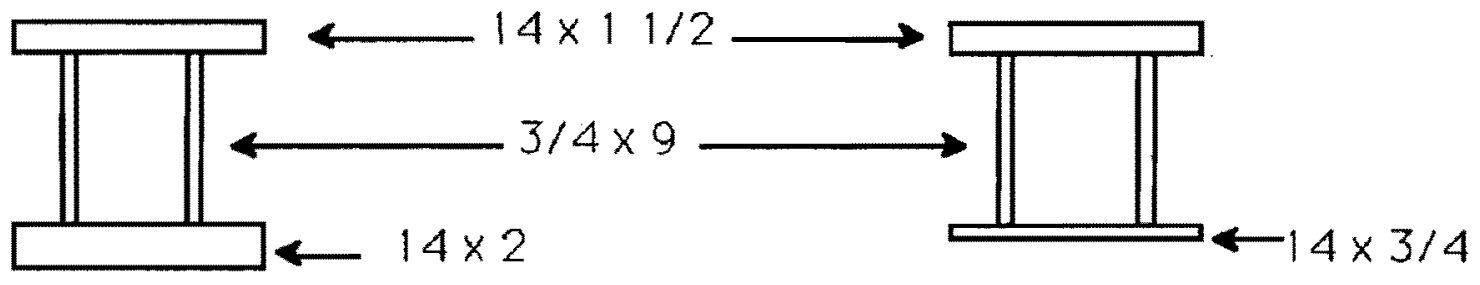

\begin{tabular}{llllrr} 
& $A$ & $Y$ & \multicolumn{1}{c}{ Ay } & \multicolumn{1}{c}{ Ad } & \multicolumn{1}{c}{1} \\
\hline 1 & 21. & 11.75 & 246.75 & 743.5 & 3.9 \\
2 & 13.5 & 6.5 & 87.75 & 6.6 & 91.1 \\
3 & 28. & 1. & 28. & 645.1 & 9.3 \\
\hline & 62.5 & & 362.5 & 1395.2 & 104.3
\end{tabular}

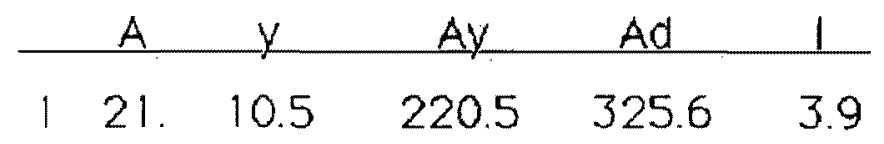

$\begin{array}{llllll}2 & 13.5 & 5.25 & 70.875 & 23.16 & 91.1\end{array}$

$\begin{array}{llllll}3 & 10.5 & .375 & 3.93 & 536.1 & .49\end{array}$

$$
y=5.8 \quad I=1500 \quad S b=258.8 \quad S t=223.8 \quad y=6.56 \quad I=981 \quad S b=149.6 \quad S t=209
$$

$M=F d=178 * 9.97=1775$

$M=36.5 * 9.97=364$

$\mathrm{fb}=\mathrm{M} / \mathrm{Sb}=1775 / 223.8=7.93$

$f b=364 / 149.6=2.43$

$f a=F \times / A=130 / 62.5=2.08$

$f a=36.5 / 45=.589$

$f s=p /(d * t)=178 /(12.5 * 1.5)=9.5$

$f 5=36.5 /(12.5 * 1.5)=1.95$ 


\section{SECTION B-B}

\section{REAR}

FRONT

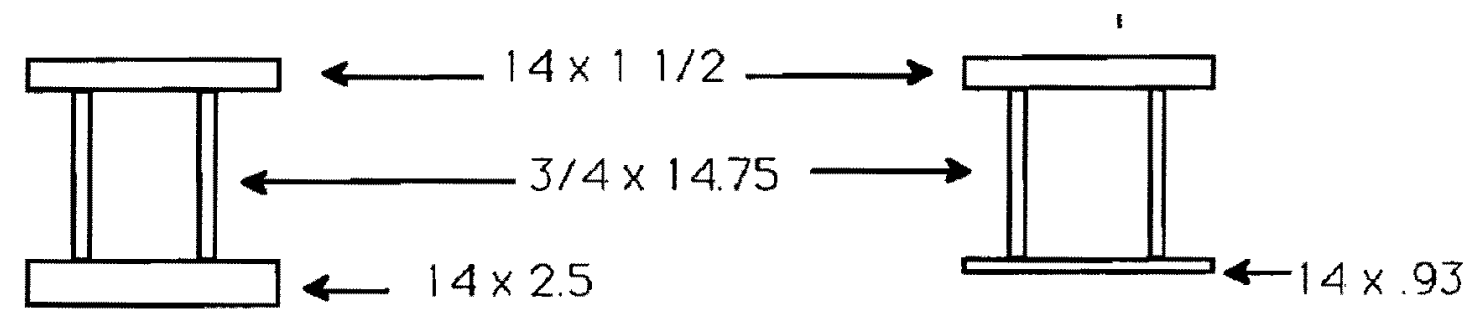

\begin{tabular}{|c|c|c|c|c|c|c|c|c|c|c|c|}
\hline & A & $y$ & Ay & $\mathrm{Ad}$ & 1 & & A & $y$ & Ay & $\mathrm{Ad}$ & 1 \\
\hline 1 & 21. & 15. & 315. & 1386. & 3.9 & 1 & 21. & 16.43 & 345. & 968. & 3.9 \\
\hline 2 & 17.63 & 8.3 & 147.6 & 40. & 401. & 2 & 17.63 & 8.3 & 146.4 & 31.4 & 401 \\
\hline 3 & 35. & 1. 25 & 43.75 & 1108. & 18.3 & 3 & 13.02 & .465 & 6.05 & 1096 & .938 \\
\hline & 73.63 & & 506.4 & 253 & 423 & & 51.6 & & 97.5 & 2095 & 40 \\
\hline
\end{tabular}

$y=6.88 \quad I=2957 \quad S b=249 \quad S t=430 \quad y=9.64 \quad I=2501 \quad S b=260 . \quad S t=146$

$M=F * d=220 *(73.35-61.016)=2714 . \quad M=45 *(73.35-61.016)=555$.

$f b=M / S b=2714 / 249=10.9 \quad f b=555 * 146=3.8$

$f S=F /(d * t)=220 /(15.75 * 1.5)=9.31$ fS $=45 /(15.75 * 1.5)=1.9$ 


\section{SECTION B1-B1}

REAB

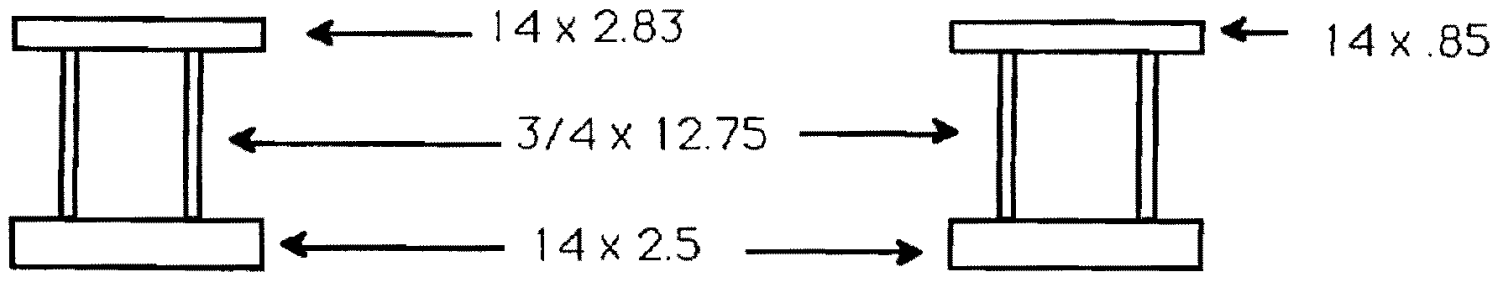

FRONT

\section{A}

$396 \quad 16.7$

2287

$A d$

39.425 .4

39.4259.

$43.752135 . \quad 18.2$

93.73

$849.254462 . \quad 303.6$

$y=9.06 \quad \mid=4766 \quad S b=526 . \quad S t=528$.

$M=F * d=220 *(73.35-50)=5135$

$f b=M / S b=5135 / 526 .=9.76$

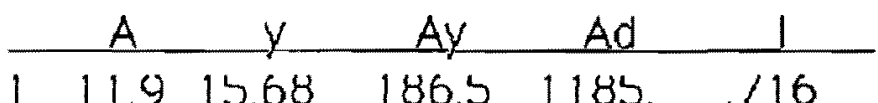
$\begin{array}{llllll}2 & 19.13 & 7.625 & 145.8 & 71 . & 259 .\end{array}$ $\begin{array}{llllll}3 & 35 & 1.25 & 43.75 & 692 & 18.2\end{array}$
376. 1948.278.

$y=5.7 \quad I=2226 \quad S b=391 \quad S t=180$.

$M=45 *(73.35-50)=1050.3$

$\mathrm{fb}=1050 / 180=5.8$ 


\section{SECTION B2-B2}

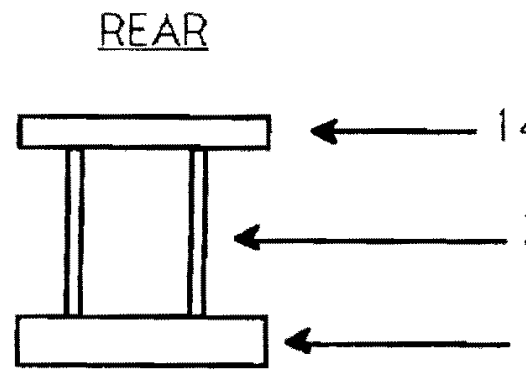

$14 \times 2.75$ $3 / 4 \times 13.75$

$14 \times 2.5$

\section{FRONT}

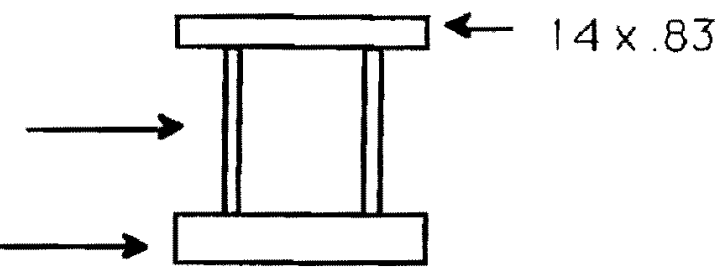

$\begin{array}{ccccc}A & y & \text { Ay } & \text { Ad } & 1\end{array}$

$\begin{array}{llllll}1 & 38.5 & 17.63 & 678.6 & 2562 . & 24.3\end{array}$

$\begin{array}{llllll}2 & 20.63 & 8.13 & 167.6 & 37.2325 .\end{array}$

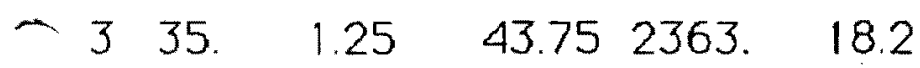

94. $889.9 \quad 4962 . \quad 367.5$

$y=9.47 \quad I=5330 \quad S b=563 \quad S t=559$

$M=F * d=220 *(73.35-45)=6237$

$f b=M / S b=6237 / 559=11.16$

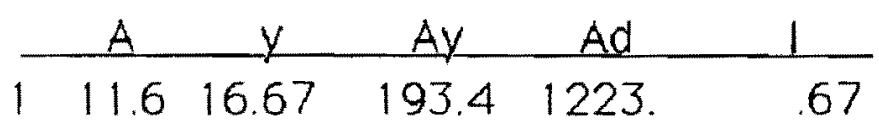

$\begin{array}{llllll}2 & 20.6 & 9.375 & 193 . & 182 . & 325 .\end{array}$

$\begin{array}{llllll}3 & 35 . & 1.25 & 43.75 & 929 . & 18.2\end{array}$

$\begin{array}{llll}67.2 & 430.1 & 2334 . & 344 .\end{array}$

$y=6.4 \quad l=2678 \quad S b=418 \quad S t=251$.

$M=45 *(73.35-45)=1275$.

$f b=1275 / 251=5.08$ 


\section{SECTION B3-B3}

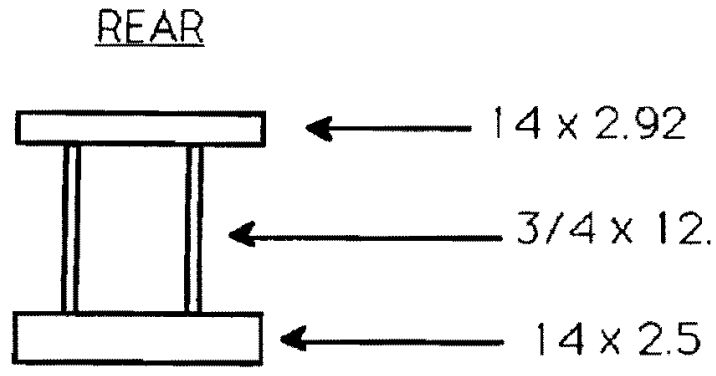

\section{FRONT}

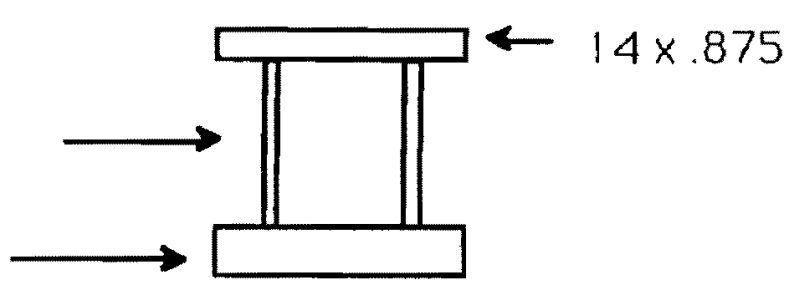

A $\quad y$

Ay

$\mathrm{Ad}$

1

$\begin{array}{llllll}1 & 48.9 & 15.96 & 652.4 & 2852 . & 29 .\end{array}$

$-$

218

8.5

153.

.56216 .

A

y

Ay $\quad A d$

1

$\begin{array}{llllll}1 & 12.25 & 14.94 & 183 . & 1018 & .782\end{array}$

$\begin{array}{llllll}2 & 18 & 8.5 & 153 . & 129 . & 216 .\end{array}$

$\begin{array}{llllll}3 & 35 . & 1.25 & 43.75 & 1750 . & 18.2\end{array}$

102.

849. 4604. 263.

$y=8.32 \quad l=5022 \quad 5 b=604 \quad 5 t=552$

$y=5.82 \quad l=2113 \quad 5 b=363 \quad S t=221$.

$M=F * d=220 *(73.35-55)=4037$

$M=45 *(73.35-55)=825.75$.

$\mathrm{fb}=M / S b=4037 / 552=7.3$

$\mathrm{fb}=825.75 / 221=3.74$ 


\section{SECTION B4-B4}

REAR

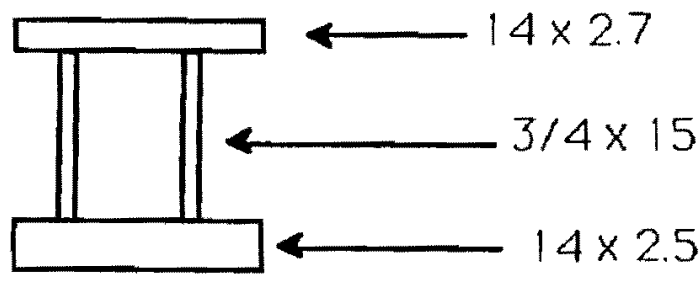

FRONT

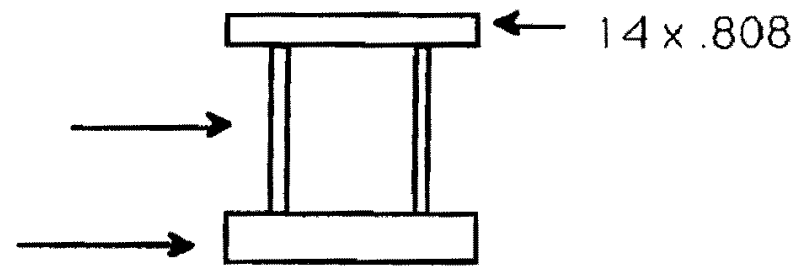

A $y$ Ay Ad 1

$\begin{array}{llllll}1 & 37.8 & 18.85 & 712.5 & 2766 . & 22.9\end{array}$

$\begin{array}{llllll}-2 & 22.5 & 10 . & 225 & 1.98 & 422 .\end{array}$

$\begin{array}{llllll}3 & 35 . & 1.25 & 43.75 & 2363 . & 18.2\end{array}$

95.3981 .255632 .463$.

$y=10.296 \quad I=6095 \quad S b=592 \quad S t=615$

$M=F * d=220 *(73.35-40)=7337$

$f b=M / S b=7337 / 592=12.4$

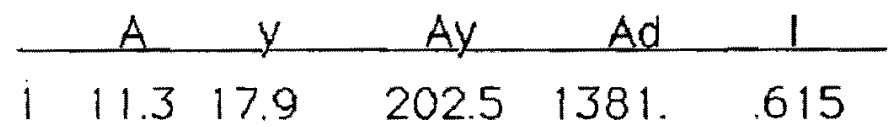

$\begin{array}{llllll}2 & 22.5 & 10 . & 225 . & 223.8 & 422 .\end{array}$

$\begin{array}{llllll}3 & 35 . & 1.25 & 43.75 & 929 . & 18.2\end{array}$

$\begin{array}{llll}68.8 & 471 & 2700.8 & 440.8\end{array}$

$y=6.85 \quad I=3142 \quad S b=459 \quad S t=274$.

$M=45 *(73.35-40)=1500.75$

$f b=1501 / 274=5.5$ 


\section{SECTION B5-BS}

REAR

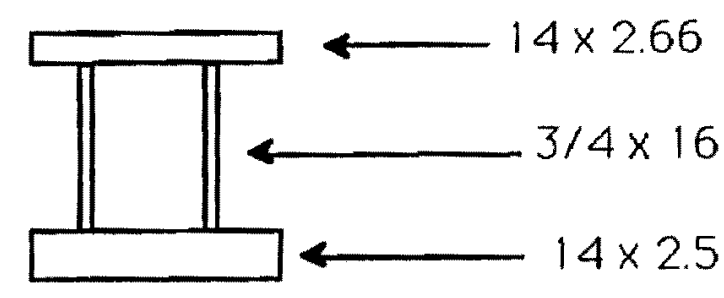

$\mathrm{Ad}$

Ay

$-$

$\begin{array}{llllll}1 & 37.24 & 19.83 & 738.5 & 3072.6 & 21.96\end{array}$

$224 . \quad 10.5 \quad 252 . \quad 1.459512$.

$\begin{array}{lllll}3 & 35 . & 1.25 & 43.753156 . & 18.2\end{array}$

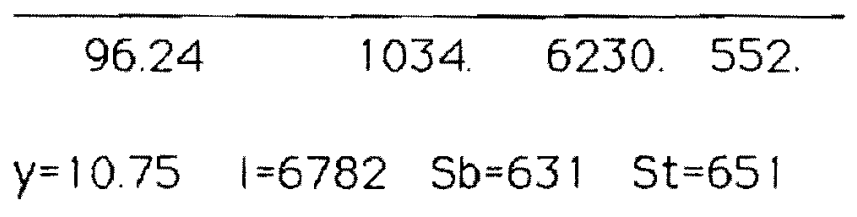

$$
\begin{aligned}
& M=F * d=220 *(73.35-36.43)=8122.4 \\
& f b=M / S b=8122.4 / 631=12.87
\end{aligned}
$$

\section{FRONT}

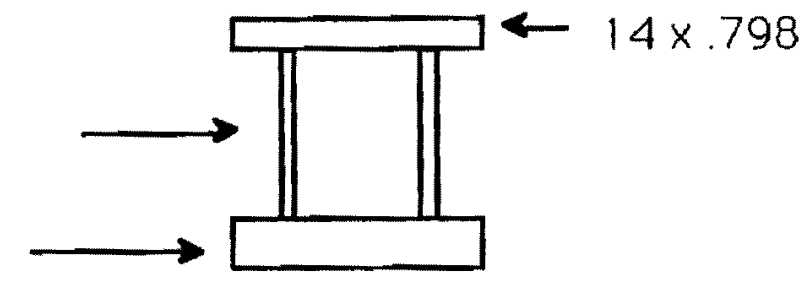

\begin{tabular}{llllcc} 
& $A$ & $y$ & Ay & Ad & 1 \\
\hline 1 & 11.17 & 18.9 & 211. & 1524 & .593 \\
2 & 24. & 10.5 & 252. & 258.2 & 512. \\
3 & 35. & 1.25 & 43.75 & 1247. & 18.2
\end{tabular}

70.172

$506.9 \quad 3029.6 \quad 530.8$

$y=7.22 \quad I=3560 \quad S b=493 \quad S t=295$.

$M=45 *(73.35-36.43)=1661.4$.

$f b=1661.4 / 295=5.63$ 


\section{SECTION D-D}

REAR

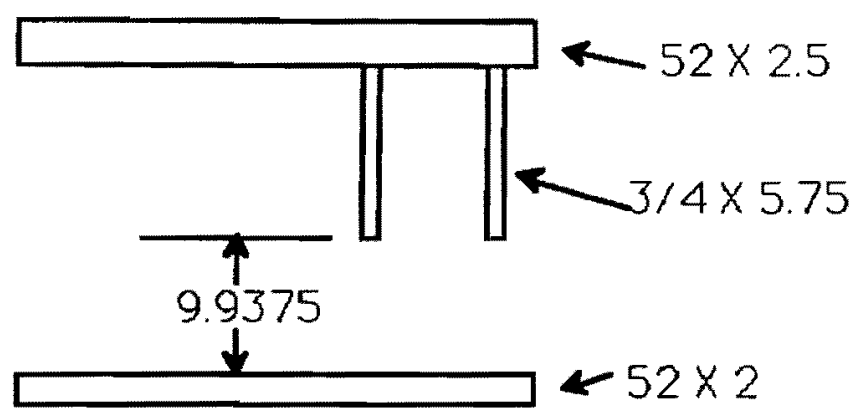

\section{FRONT}

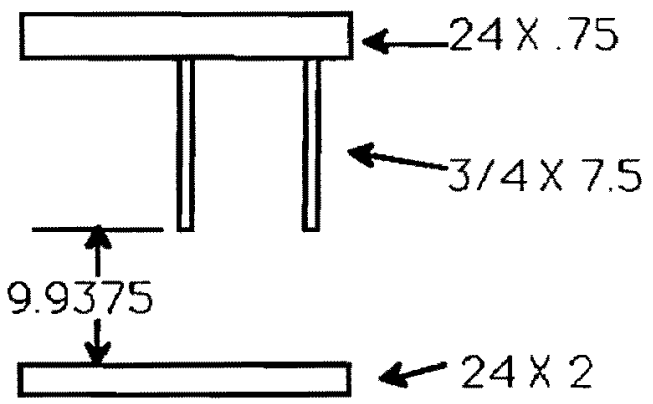

$\begin{array}{lllll}A & y & A y & A d & 1\end{array}$

$\begin{array}{llllll}1 & 130 . & 18.94 & 2462 . & 7978 . & 67.7\end{array}$ $\begin{array}{llllll}2 & 8.63 & 14.813 & 127.76 & 118.6 & 27.76\end{array}$ $\begin{array}{llllll}3 & 104.104 & 10 & 10617 . & 34.67\end{array}$

242.6 2693. 18713. 130.

$y=11.1 \quad l=18843 \quad S b=1697 \quad S t=2075$

$M=F * d=220 *(73.35-45)=6237$

$f b=M / S b=6237 / 559=11.16$

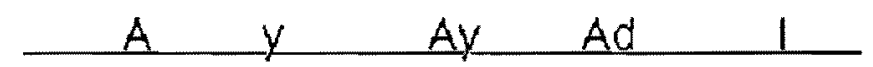

$\begin{array}{llllll}1 & 18 . & 19.813 & 356.6 & 2719 . & .844\end{array}$ $\begin{array}{llllll}2 & 11.25 & 15.688 & 176.5 & 750.52 .73\end{array}$ $348.1 .48 .2042,16$. $y=7.52 \quad I=5581 \quad S b=742 \quad S t=441$.

$M=45 *(73.35-45)=1275$.

$f b=1275 / 251=5.08$ 


\section{SECTION E-E}
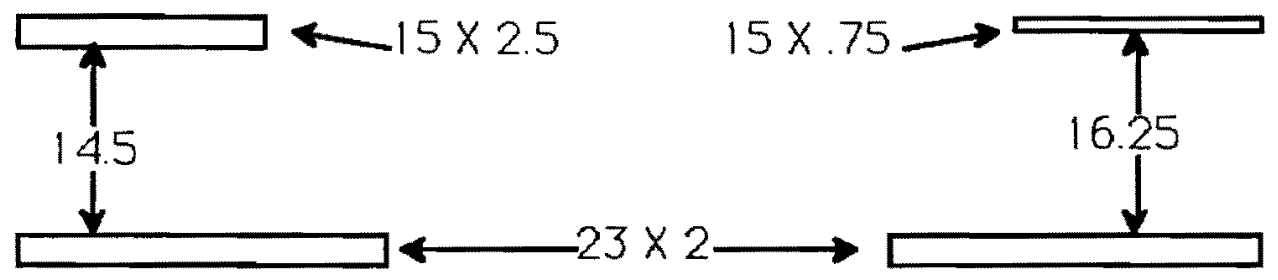

$$
\begin{aligned}
& -M=220 *(73.34-21.875)=11322 \quad M=45 *(73.34-21.875)=2316 \\
& T=11322 / 17.75=638 \\
& T=316 / 17.75=124 \\
& \mathrm{ft}=T /(\mathrm{t} * \mathrm{~L})=638 /(2.5 * 15)=17 \quad \mathrm{ft}=124 /(.75 * 15)=11 \\
& f C=T /(t * L)=638 / 2 * 23)=13.9 \quad f C=124 /(2 * 23)=2.7
\end{aligned}
$$




\section{STANCHION SUPPORT PLATE}

2" ANGLED PLATE

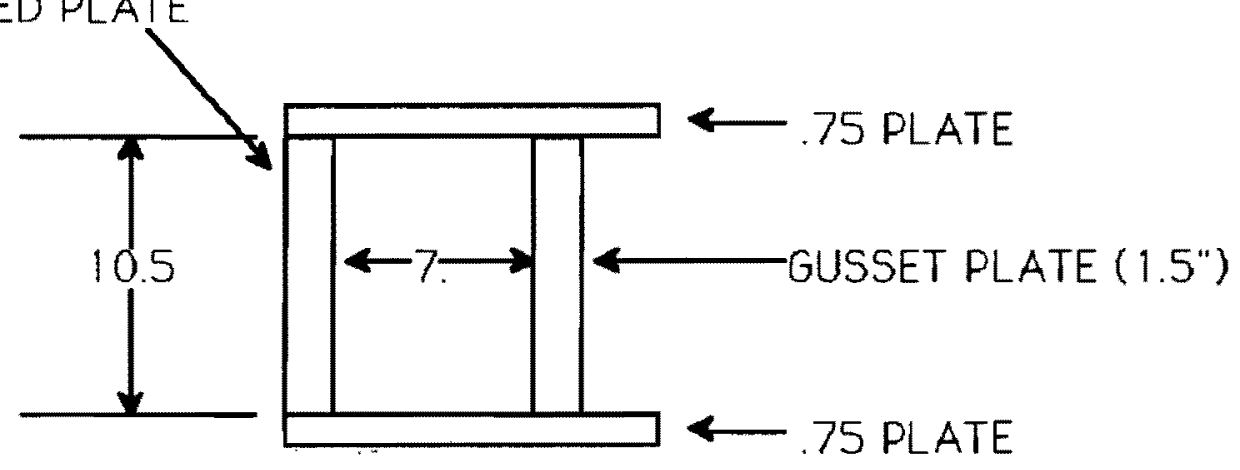

TOP VIEW

AREA OF LOAD $=13^{\prime \prime}$ CIRCLE

$P=F / A=220 /(3.14 * 6.5 * 6.5)=1.66$

IF THE LOAD IS CENTERED OVER THE GUSSET PLATE, HALF THE LOAD IS OVER THE PLATE AREA BETWEEN THE ANGLES PLATE AND GUSSET PLATE

PER ROARK AND YOUNG, TABLE 26, CASE la FOR A PLATE $10.5 \times 7$ :

$a / b=10.5 / 7=1.5 \quad B=.7116$

$f b=(B *(P / 2) * b * b) /(t * t)=(.7116 * .83 * 7 * 7) /(1.5 * 1.5)=12.9$ 


\section{STANCHION GUSSET PLATE}
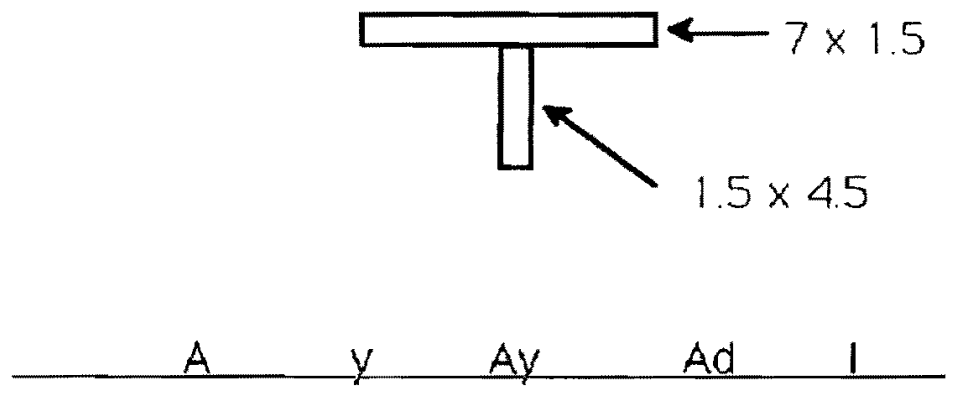

\begin{tabular}{cccccc}
1 & 10.5 & 5.25 & 55.125 & 14.5 & 1.969 \\
2 & 6.75 & 2.25 & 15.188 & 22.5 & 11.4 \\
\hline
\end{tabular}

$$
\begin{aligned}
& \begin{array}{llll}
17.25 & 70.313 \quad 37 & 13.36
\end{array} \\
& y=4.076 \quad 1=50.36 \quad \text { Sb }=12.4 \quad \text { St }=26.2 \\
& w=110 / 10.5=10.5 \\
& M=(w * L * L) / 8=(10.5 * 10.5 * 10.5) / 8=144 \\
& \mathrm{fb}=\mathrm{M} / \mathrm{S}=144 / 12.4=11.7
\end{aligned}
$$




\section{SUPPORT}

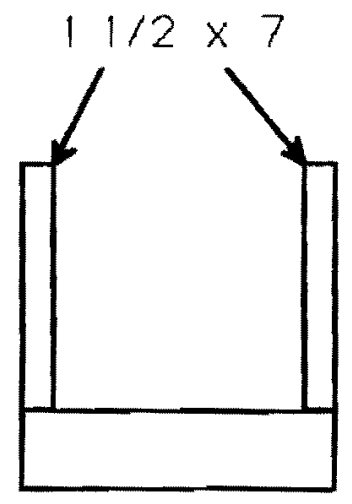

$4 \times 161 / 4$

\begin{tabular}{rrrrrrr}
1 & 21. & 19.25 & 414.75 & 1442 & 85.75 \\
2 & 65. & 8.13 & 528.13 & 524 & 86.67 \\
\hline & 86. & & 942.88 & 1966 & 172.
\end{tabular}

$y=10.96 \quad l=2138 . \quad S b=195 . \quad S t=174$.

Max. Moment accurs when load is at the edge $(1=10)$

$\operatorname{Mmax}=10 \times 220=2200$

$f b=2200 / 174=12.6$ 


\section{BEARING PLATE}

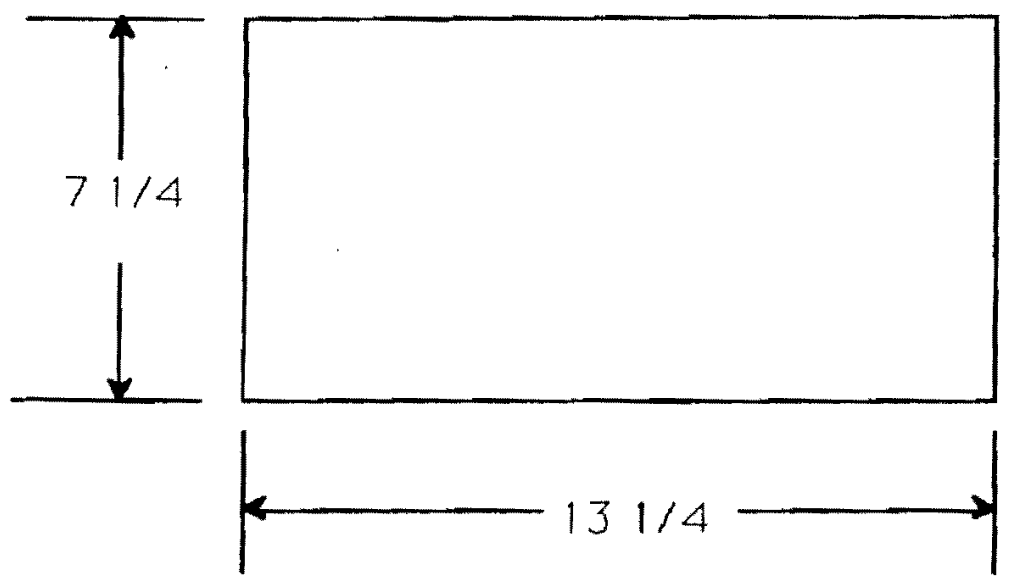

Per Roark, Table 26 \#f

$f b=(B 2 * w * b) /(t * t)$

$a=131 / 4 \quad b=71 / 4 \quad B 2=1.788$

$w=220 / 13.25=16.7$

$f b=(1.788 * 16.7 * 7.25) /(4 * 4)=13.45$ 


\section{WELD CALCULATIONS}

$$
W=(V * A * y) /(n * 1 * 12)
$$

\section{SECIIION A-A}

TOP

BOTTOM
$V=178 \quad A=14 * 1.5=21$
$V=178 \quad A=14 * 2=28$
$y=11.75-5.8=5.95$
$y=5.8-1=4.8$
$1=1500$
$1=1500$
$w=.62$
$w=.66$

\section{SECTION B-B}

TOP

BOTTOM

$A=14 * 1.5=21$

$A=14 * 2.5=35$

$y=15-6.88=8.12$

$y=6.88-1=5.88$

$v=220 \quad 1=2957$

$V=220 \quad 1=2957$

$w=53$

$w=61$ 
SECTION B5-B5

TOP

\section{BOTTOM}

$A=14 * 2.66=37.24$

$A=14 * 2.5=35$.

$y=19.83-10.75=9.08$

$y=10.75-1.25=9.5$

$V=220 \quad 1=6782$

$V=220 \quad \mid=6782$

$W=.46$

$w=.45$

SECTIONC-C

TOP

BOTTOM

$A=18 * 2.6=46.8$

$A=18 * 2=36$

$y=23.1-13.07=10.03$

$y=13.07-1=12.07$

$V=110 \quad I=11001$

$V=110 \quad 1=11001$

$W=.196$

$W=.18$ 


\section{SECTIOND-D}

$$
\begin{aligned}
& A=52 * 2.5=130 \\
& Y=18.94-11.1=7.84 \\
& V=110 \quad 1=18843 \\
& W=.25
\end{aligned}
$$




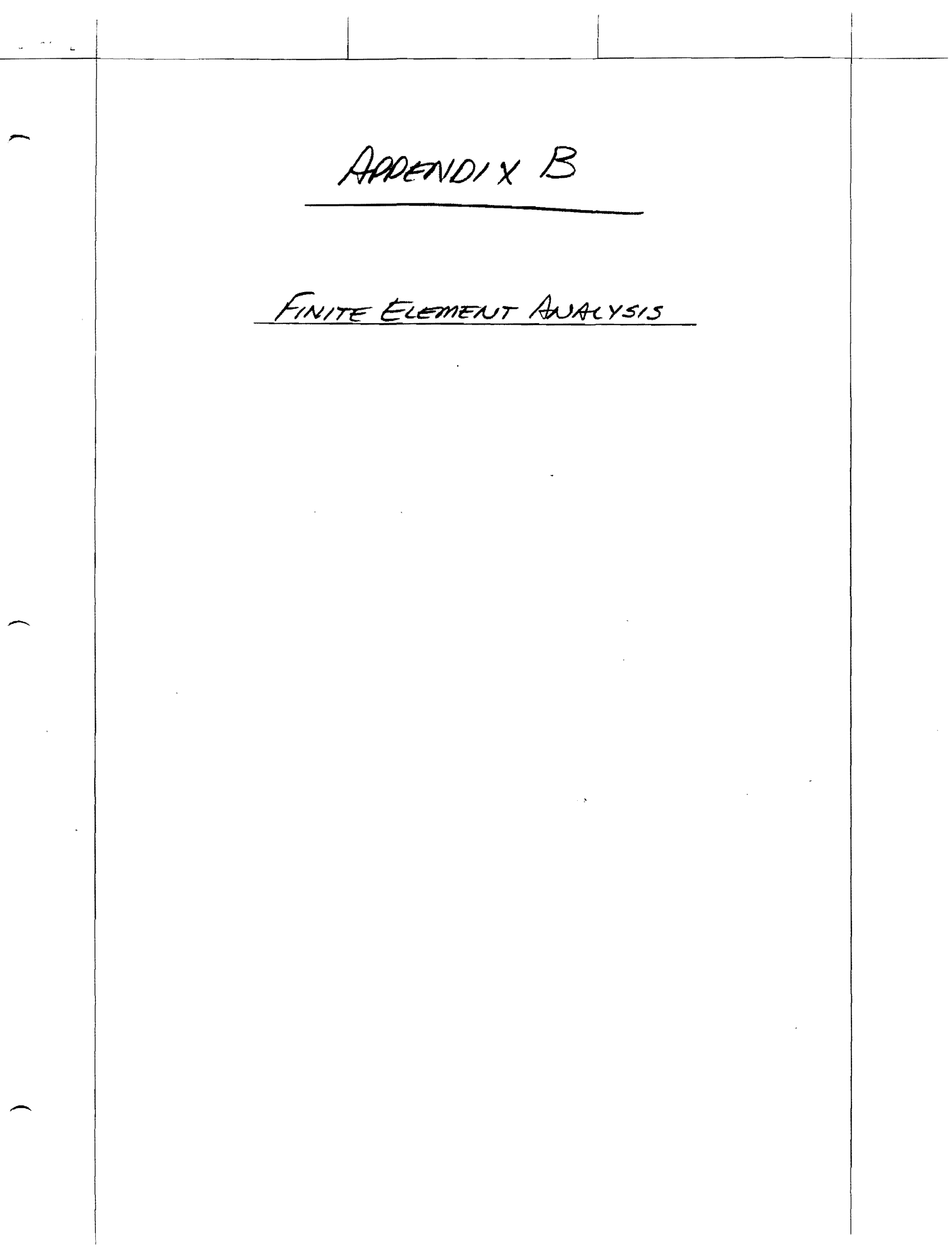




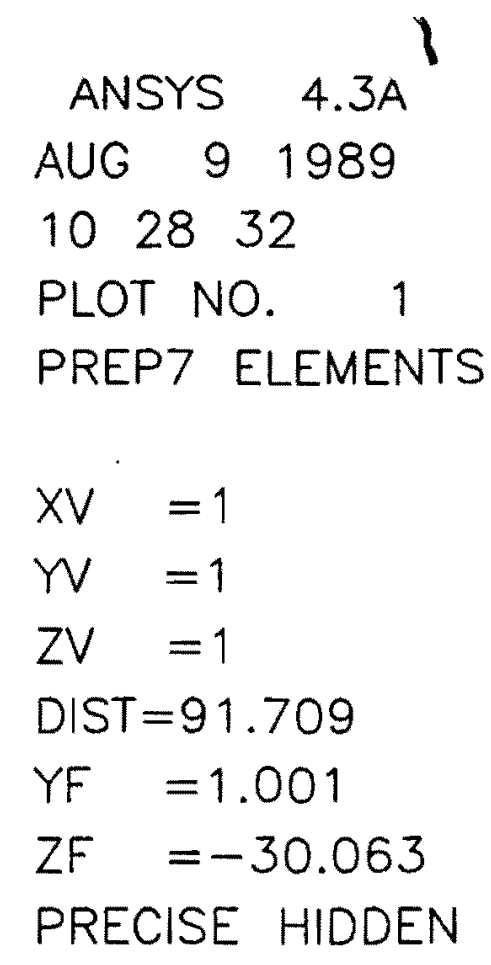

